\title{
Optimal Stealth Trajectory Design to Deceive Anomaly Detection Process
}

\author{
Enrica d'Afflisio*, Augusto Aubry ${ }^{\dagger}$, Paolo Braca*, Antonio De Maio ${ }^{\dagger}$, and Leonardo M. Millefiori* \\ * NATO STO Centre for Maritime Research and Experimentation, La Spezia, Italy \\ $\dagger$ Department of Electrical and Information Technology Engineering, University of Naples Federico II, Napoli, Italy
}

\begin{abstract}
A new methodology is proposed to deceive an anomalous trajectory detector by designing ship paths that deviate from the nominal traffic routes in an optimized way. The route planning is formalized as a min-max problem (with respect to surveillance system acquisition instants) focusing on the Kullback-Leibler (KL) divergence between the statistical hypotheses of the nominal and the anomalous trajectories as key performance measure. Modeling the vessel's dynamic according to the Ornstein-Uhlenbeck $(\mathrm{OU})$ mean-reverting stochastic process, physical, practical, and kinematic requirements are also accounted for forcing several constraints at the design stage. A computationally efficient technique is proposed to handle the resulting non-convex optimization problem, and some case studies are reported to assess its effectiveness.

Index Terms-Ornstein-Uhlenbeck process, maritime anomaly detection, statistical hypothesis test, target tracking, automatic identification system, maritime security, real-world data.
\end{abstract}

\section{INTRODUCTION}

Maritime Situational Awareness (MSA) is an important domain for many national and international institutions, agencies and bodies. MSA provides support by responding to the needs of a wide range of maritime policies as irregular migration, border control, maritime security, fisheries control, anti-piracy, oil pollution, smuggling and terrorism. MSA requires information from a combination of heterogeneous sensor systems, such as radars, Synthetic Aperture Radar (SAR), video and infrared cameras, and the self-reporting Automatic Identification System (AIS) [1]. AIS devices are used by ships to regularly broadcast data about their identity, position, velocity, course, destination, and other details at predefined intervals.

The analysis of recorded AIS data can be used to identify recurrent traffic patterns [2], [3]; this is possible because most of the ships, especially in open sea, seek to optimize fuel consumption, and therefore tend to maneuver very seldom, at the same time keeping their speed as much constant as possible. If a ship exhibits a pattern differing from such expected one, it can be declared to have an anomalous behavior, indicating possible illicit activities. From a more practical perspective instead, operators manually search and try to predict critical situations, such as potential collisions and suspicious activities performed by many vessels within vast sea areas. In order to provide support in these operations, a number of methods and systems with anomaly detection capabilities have been proposed [4]. Indeed, over the past few years, anomaly detection strategies have been applied to maritime traffic monitoring [2], [3], [5]-[9], to detect, e.g., unexpected stops or course changes (path deviations), and, more generally, any vessel's anomalous behavior that might be related to potential suspicious activity. Most of previous approaches [2], [3], [5], [6] consist in extracting maritime traffic patterns by mining of historical data, and detecting anomalous vessel behavior by using unsupervised learning techniques. More recently, a novel maritime anomaly detector [8], [9], has been proposed to reveal possible path deviations during an intentional disablement of the AIS transponder or during a period without data available, relying only on the available measurements. Specifically, this detector is based on a hypothesis testing procedure able to identify changes in the longrun mean velocity parameter of the Ornstein-Uhlenbeck (OU) process, which was shown [10]-[12] to be a realistic model for vessels' dynamics in open sea.

Building on the detection strategy presented in [8], [9], this work proposes a new methodology to design the optimal stealth trajectory of a vessel aiming to deviate from a nominal traffic route, so as to reach a specific location point, where, for instance, a rendezvous with another ship (for some possible illegal exchanges) has to occur [13]. To maliciously hide this task, the vessel turns off its AIS transponder for the time sufficient to arrive to the specific point, stop there, and then go back to the expected route. In addition, it is assumed that, during the AIS device shutdown, the vessel can only be observed by satellite-based sensors, such as SAR, whose sequence of possible sensor snapshot positions is supposed known to the vessel.

The proposed route planning problem aimed at optimally defining the ship trajectory is formalized as a constrained optimization problem, where physical requirements on the mean velocity dynamic, the compliance of the trajectory with the underlying OU statistical model, the existence of a time instant allowing the rendezvous, as well as sea coast impairments are accounted for at design stage. An efficient solution technique is proposed to handle the derived non-convex optimization problem. Remarkably, leveraging the proposed optimization tool, it is possible to determine optimized surveillance instants to improve the anomaly detector capability. Interesting case studies are finally reported to prove the effectiveness of the proposed planning strategy.

The paper is structured as follows: Section II briefly describes the maritime scenario, the OU model and the anomaly 
detector [8], [9]; in Section III the route planning strategy is formalized; the effectiveness of the proposed methodology is shown through the performance analysis in Section IV, applied to both synthetic and real-world scenarios. Finally, concluding remarks and possible future research lines are summarized in $\mathrm{V}$.

\section{NOTATION}

We use boldface for vectors $\boldsymbol{a}$ (lower case), and matrices $\boldsymbol{A}$ (upper case). The transpose operator is denoted by the symbols $(\cdot)^{\mathrm{T}} . \boldsymbol{I}$ and $\mathbf{0}$ denote respectively the identity matrix and matrix with zero entries (their size is determined by the context). $\mathbb{R}^{N}, \mathbb{S}^{N}$ and $\mathbb{S}_{++}^{N}$ are respectively the sets of $N$ dimensional vectors of real numbers, of $N \times N$ symmetric matrices, and of $N \times N$ positive definite symmetric matrices. The curled inequality symbol $\succeq$ (and its strict form $\succ$ ) is used to denote generalized matrix inequality: for any $\boldsymbol{A} \in \mathbb{S}^{N}$, $\boldsymbol{A} \succeq \mathbf{0}$ means that $\boldsymbol{A}$ is a positive semi-definite matrix ( $\boldsymbol{A} \succ \mathbf{0}$ for positive definiteness). $\|\boldsymbol{x}\|_{\boldsymbol{A}}$ denotes the Mahalanobis norm induced by the positive definite matrix $\boldsymbol{A}$. The standard Euclidean norm is indicated with $\|\boldsymbol{x}\|$. Besides, for any set $\mathcal{A},|\mathcal{A}|$ represents the cardinality of $\mathcal{A}$. Finally $\mathbb{E}[\cdot]$ denotes statistical expectation, and, for any optimization problem $\mathcal{P}$, $v(\mathcal{P})$ represents its optimal value.

\section{Maritime Anomaly Detection}

\section{A. Maritime anomaly scenario}

We consider the situation where a vessel is following a planned trajectory with a nominal velocity $\boldsymbol{v}_{0}$, and at some point it turns its AIS device off for a certain time period $T$, in order to hide an unexpected deviation from the nominal behavior or simply the communication is interrupted. In particular, in this work, we focus on the specific event that, in the period of disablement of the AIS transponder, the vessel loiters for some time in a targeted location, possibly to meet another ship, even if the case of a generic anomalous trajectory can be easily handled by the anomaly detection strategy proposed in [8]. At the end of the period T, the AIS device is switched back on, and the vessel restores its nominal navigating conditions. More details are provided in [8] about the aforementioned maritime anomaly detection.

During the silent period, the vessel is assumed to move according to a piecewise OU model with a sequence of $N$ unknown long-run mean velocities, comprised in the matrix $\boldsymbol{V}=\left[\boldsymbol{v}_{1}, \boldsymbol{v}_{2}, \ldots \boldsymbol{v}_{N}\right] \in \mathbb{R}^{2, N}$, which identifies an $N$-section path. The nominal vessel's positions along this path, corresponding to each junction point between consecutive segments, are enclosed in the matrix $\boldsymbol{P}=\left[\boldsymbol{p}_{0}, \boldsymbol{p}_{1}, \boldsymbol{p}_{2}, \ldots \boldsymbol{p}_{N}\right] \in \mathbb{R}^{2, N+1}$. The time taken to cover the single section corresponds to the time interval $\left[t_{n-1}, t_{n}\right]$, with $n=1, \ldots, N$, which clearly depends on the aforementioned quantities.

\section{B. Ornstein-Uhlenbeck model}

First of all, the OU model characterized by a single long-run mean velocity parameter is introduced, and then its extension to a piecewise-constant long-run mean velocity is provided.
To begin, let us indicate the four-dimensional target state at time $t \in \mathbb{R}_{0}^{+}$with

$$
\boldsymbol{s}(t)=[\boldsymbol{p}(t), \dot{\boldsymbol{p}}(t)]^{\mathrm{T}},
$$

where $\boldsymbol{p}(t)$ and $\dot{\boldsymbol{p}}(t)$ denote the target position and velocity, respectively, in a two-dimensional Cartesian reference system

$$
\boldsymbol{p}(t)=[x(t), y(t)], \dot{\boldsymbol{p}}(t)=[\dot{x}(t), \dot{y}(t)] .
$$

The target dynamics, in general, can be modeled with a set of linear stochastic differential equations (SDEs) [14], and in [10] it is shown how the movement of real non-maneuvering vessels in the open sea can be represented by a mean-reverting stochastic process. Specifically, the velocity of the target is an OU process, and its position is an Integrated OU (IOU) process. Under this assumption, the SDE for the target motion model has the following form

$$
\mathrm{d} \boldsymbol{s}(t)=\boldsymbol{A} \boldsymbol{s}(t) \mathrm{d} t+\boldsymbol{G} \boldsymbol{v} \mathrm{d} t+\boldsymbol{B} \mathrm{d} \boldsymbol{\omega}(t),
$$

where $\boldsymbol{v}=\left[\begin{array}{ll}v_{x} & v_{y}\end{array}\right]^{\mathrm{T}}$ is the long-run process mean, and $\boldsymbol{\omega}(t)$ is a standard bi-dimensional Wiener process. The matrices $\boldsymbol{A}$, $\boldsymbol{B}$ and $\boldsymbol{G}$ are defined as:

$$
\boldsymbol{A}=\left[\begin{array}{cc}
\mathbf{0} & \boldsymbol{I} \\
\mathbf{0} & -\boldsymbol{\Theta}
\end{array}\right], \quad \boldsymbol{B}=\left[\begin{array}{c}
\mathbf{0} \\
\boldsymbol{\Sigma}
\end{array}\right], \quad \boldsymbol{G}=\left[\begin{array}{c}
\mathbf{0} \\
\boldsymbol{\Theta}
\end{array}\right],
$$

where $\mathbf{0}$ is a $2 \times 2$ null matrix, $\boldsymbol{\Sigma}$ is a $2 \times 2$ matrix defining the noise process and $\Theta$ is a $2 \times 2$ matrix quantifying the meanreversion effect, meaning the rate at which the target will tend back to the desired velocity after a perturbation; its diagonal terms refer to the $x$ and $y$ components, while the off-diagonals quantify the coupling effect.

Model (3) is only able to describe a non-maneuvering target, i.e., ships whose long-run mean velocity does not change in time. However, the model can be extended to the case of waypoint navigation [15], that is relevant to our application. In this respect, the long-run mean velocity of the target can be assumed as a piecewise-constant function of the time that takes values from a sequence $\boldsymbol{v}_{1}, \ldots, \boldsymbol{v}_{N}$. Along the navigational legs, the long-run mean velocity of the process is constant and the instantaneous velocity of the target follows, by all means, an OU process; conversely, the maneuver is represented by a change of the long-run mean velocity parameter. To proceed further in describing the piecewise OU model, unless otherwise stated, we will use hereafter sub-scripted indices to denote discrete-time dependency, i.e. $\boldsymbol{p}_{n}=\boldsymbol{p}\left(t_{n}\right), \dot{\boldsymbol{p}}_{n}=\dot{\boldsymbol{p}}\left(t_{n}\right)$ and $s_{n}=\boldsymbol{s}\left(t_{n}\right)$ by definition. In this way, the target state at time $t_{n}$, given the target state at the previous time $t_{n-1}$, can be written in matrix form as

$$
\boldsymbol{s}_{n}=\boldsymbol{\Phi}\left(t_{n}-t_{n-1}, \boldsymbol{\gamma}\right) \boldsymbol{s}_{n-1}+\boldsymbol{\Psi}\left(t_{n}-t_{n-1}, \boldsymbol{\gamma}\right) \boldsymbol{v}_{n}+\boldsymbol{\omega}_{n}
$$

where $\boldsymbol{v}_{n}$ is the long-run mean velocity in the time interval $\Delta t_{n}=\left[t_{n-1}, t_{n}\right]$ and $\boldsymbol{\omega}_{n}$ is a zero-mean Gaussian random variable with covariance $\boldsymbol{C}\left(\Delta_{n}\right)$ defined in [8]. The state transition matrix and the control input function, $\boldsymbol{\Phi}\left(\Delta t_{n}, \gamma\right)$ and $\boldsymbol{\Psi}\left(\Delta t_{n}, \boldsymbol{\gamma}\right)$, respectively, are defined as

$$
\boldsymbol{\Phi}\left(\Delta t_{n}, \boldsymbol{\gamma}\right)=\left[\begin{array}{cc}
\boldsymbol{I} & \left(\boldsymbol{I}-e^{-\boldsymbol{\Gamma} \Delta t_{n}}\right) \boldsymbol{\Gamma}^{-1} \\
\mathbf{0} & e^{-\boldsymbol{\Gamma} \Delta t_{n}}
\end{array}\right]
$$




$$
\boldsymbol{\Psi}\left(\Delta t_{n}, \gamma\right)=\left[\begin{array}{c}
\Delta t_{n} \boldsymbol{I}-\left(\boldsymbol{I}-e^{-\boldsymbol{\Gamma} \Delta t_{n}}\right) \boldsymbol{\Gamma}^{-1} \\
\boldsymbol{I}-e^{-\boldsymbol{\Gamma} \Delta t_{n}}
\end{array}\right] .
$$

The target state at time $t_{N}$ can be expressed recursively as (omitting $\gamma$ for clarity)

$$
\begin{aligned}
\boldsymbol{s}_{N}= & \boldsymbol{\Phi}\left(\Delta_{N}\right) \boldsymbol{s}_{N-1}+\boldsymbol{\Psi}\left(\Delta_{N}\right) \boldsymbol{v}_{N}+\boldsymbol{\omega}_{N} \\
= & \boldsymbol{\Phi}\left(\Delta_{N}\right)\left[\boldsymbol{\Phi}\left(\Delta_{N-1},\right) \boldsymbol{s}_{N-2}+\boldsymbol{\Psi}\left(\Delta_{N-1}\right) \boldsymbol{v}_{N-1}+\boldsymbol{\omega}_{N-1}\right] \\
& +\boldsymbol{\Psi}\left(\Delta_{N}\right) \boldsymbol{v}_{N}+\boldsymbol{\omega}_{N} \\
= & \boldsymbol{\Phi}(T) \boldsymbol{s}_{0}+\boldsymbol{\Psi}\left(\Delta_{N}\right) \boldsymbol{v}_{N}+\boldsymbol{\omega}_{N} \\
& +\sum_{n=1}^{N-1}\left[\prod_{i=n+1}^{N} \boldsymbol{\Phi}\left(\Delta_{i}\right)\right]\left[\boldsymbol{\Psi}\left(\Delta_{n}\right) \boldsymbol{v}_{n}+\boldsymbol{\omega}_{n}\right]
\end{aligned}
$$

where we exploited the property of the state transition matrix by which $\boldsymbol{\Phi}\left(\Delta t_{1}\right) \boldsymbol{\Phi}\left(\Delta t_{2}\right) \ldots \boldsymbol{\Phi}\left(\Delta t_{n}\right)=\boldsymbol{\Phi}\left(\Delta t_{1}+\Delta t_{2}+\ldots+\right.$ $\left.\Delta t_{n}\right), \forall n=1, \ldots, N$, that can be derived by inspection from (6).

\section{Detection of stealth deviations}

Previous work [8] showed that a properly designed hypothesis testing procedure is able to detect a deviation from a nominal route, even if it was not possible to observe any AIS data during the deviation. We report hereafter the findings obtained in [8] when a stack of $M+1$ heterogeneous measurements (AIS, radar, SAR, etc.) is available during the period between $t_{0}$ and $T$, as $\left[\boldsymbol{z}_{0}, \ldots, \boldsymbol{z}_{m}, \ldots, \boldsymbol{z}_{M}\right]^{\mathrm{T}}$, where the $m$-th measurement is given by $\boldsymbol{z}_{m}=\boldsymbol{s}\left(T_{m}\right)+\boldsymbol{n}_{m}$. The measurement noise terms $\boldsymbol{n}_{m}$ are assumed to be independent and identically distributed according to a zeromean Gaussian with covariance $\boldsymbol{C}_{\boldsymbol{n}_{m}}$, and the measurement noise is independent of the OU process noise. Moreover the $m$-th measurement is available at time $T_{m}=\frac{p_{m}}{N} T$, where $p_{m}$ is, by definition, a fraction of the interval $[0, N]$, representing the time location of the contact with respect to the $N$ piecewise OU model velocities. In [8], the measurements processed to detect possible trajectory anomalies are given by $\boldsymbol{y}=\left[\boldsymbol{y}_{1}, \ldots, \boldsymbol{y}_{m}, \ldots, \boldsymbol{y}_{M}\right]^{\mathrm{T}}$, with $\boldsymbol{y}_{m}=\boldsymbol{z}_{m}-\Phi\left(T_{m}\right) \boldsymbol{z}_{0}$, in order to remove the dependence on $s\left(t_{0}\right)$. Hence, the anomaly detection problem addressed in [8] was traced back to the following Gaussian composite hypothesis testing problem:

$$
\left\{\begin{array}{l}
H_{0}: \boldsymbol{y} \sim \mathcal{N}\left(\boldsymbol{\mu}_{0}, \boldsymbol{C}\right) \\
H_{1}: \boldsymbol{y} \sim \mathcal{N}(\boldsymbol{\mu}, \boldsymbol{C})
\end{array}\right.
$$

where the null hypothesis, $H_{0}$, states that the vessel navigates according to the nominal condition, and the alternative one, $H_{1}$, that the vessel moves away from the nominal condition, when the AIS device is not active. The mean vectors under the two hypotheses are given by, respectively,

$$
\begin{aligned}
\boldsymbol{\mu}_{0} & =\boldsymbol{H} \operatorname{vec}\left(\boldsymbol{V}_{0}\right), \\
\boldsymbol{\mu} & =\boldsymbol{H} \operatorname{vec}(\boldsymbol{V}),
\end{aligned}
$$

where $\boldsymbol{V}_{0} \in \mathbb{R}^{2, N}$ is the known matrix related to the nominal velocity, which, differently from [8] (where the nominal velocity was considered constant) is assumed piecewise and known.
$\boldsymbol{H}$ is the model matrix of size $4 M \times 2 N$; in this work, it is assumed known ${ }^{1}$, and defined as

$$
\boldsymbol{H} \triangleq\left[\boldsymbol{F}_{1}, \ldots, \boldsymbol{F}_{m}, \ldots, \boldsymbol{F}_{M}\right]^{\mathrm{T}},
$$

where $\boldsymbol{F}_{m}$ is a $4 \times 2 N$ matrix whose definition is provided in ( [8] eq.22), and depends on the state transition matrices and the control input functions associated to the $m$-th contact. The covariance matrix is given by

$$
\boldsymbol{C} \triangleq\left[\begin{array}{cccc}
\boldsymbol{C}_{11} & \boldsymbol{C}_{12} & \cdots & \boldsymbol{C}_{1 M} \\
\boldsymbol{C}_{21} & \boldsymbol{C}_{22} & \cdots & \boldsymbol{C}_{2 M} \\
\vdots & \vdots & \ddots & \vdots \\
\boldsymbol{C}_{M 1} & \boldsymbol{C}_{M 2} & \cdots & \boldsymbol{C}_{M M}
\end{array}\right]
$$

where, $\forall i, j=1, \ldots, M$, see ( [8], Appendix C)

$$
\boldsymbol{C}_{i j}= \begin{cases}\boldsymbol{C}\left(T_{i}\right)+\boldsymbol{C}_{\mathbf{n}_{i}}+\boldsymbol{\Phi}\left(T_{i}\right) \boldsymbol{C}_{\mathbf{n}_{0}} \boldsymbol{\Phi}\left(T_{i}\right)^{\mathrm{T}} & \text { if } i=j \\ \boldsymbol{C}\left(T_{i}\right) \boldsymbol{\Phi}\left(T_{j}-T_{i}\right)^{\mathrm{T}}+\boldsymbol{\Phi}\left(T_{i}\right) \boldsymbol{C}_{\mathbf{n}_{0}} \boldsymbol{\Phi}\left(T_{j}\right)^{\mathrm{T}} & \text { if } i<j\end{cases}
$$

and $\boldsymbol{C}_{j i}=\boldsymbol{C}_{i j}^{\mathrm{T}}$.

Following the approach in [8], a Generalized Likelihood Ratio Test (G-LRT) is exploited to decide, once that the AIS device of the ship has been turned back on, whether it had been moving accordingly to the nominal route or not. Specifically, assuming $\boldsymbol{H}$ a full rank matrix, the G-LRT has the following form:

$$
\left(\widehat{\boldsymbol{v}}-\operatorname{vec}\left(\boldsymbol{V}_{0}\right)\right)^{\mathrm{T}} \boldsymbol{H}^{\mathrm{T}} \boldsymbol{C}^{-1} \boldsymbol{H}\left(\widehat{\boldsymbol{v}}-\operatorname{vec}\left(\boldsymbol{V}_{0}\right)\right) \underset{H_{0}}{\stackrel{H_{1}}{\gtrless}} \tau,
$$

where $\widehat{\boldsymbol{v}}$ is the ML estimate of $\operatorname{vec}(\boldsymbol{V})$, given by

$$
\widehat{\boldsymbol{v}}=\left(\boldsymbol{H}^{\mathrm{T}} \boldsymbol{C}^{-1} \boldsymbol{H}\right)^{-1} \boldsymbol{H}^{\mathrm{T}} \boldsymbol{C}^{-1} \boldsymbol{y},
$$

The test statistics under the two hypotheses, $H_{0}$ and $H_{1}$, are characterized, respectively, by central and non-central Chisquared distributions, both with $d=2 N$ degrees of freedom, corresponding to the size of the known parameter $\operatorname{vec}\left(\boldsymbol{V}_{0}\right)$. The detector performance is then given by

$$
P_{\mathrm{FA}}=Q_{\chi_{d}^{2}}(\tau), \quad P_{\mathrm{D}}=Q_{\chi_{d}^{\prime 2}(\lambda)}(\tau),
$$

with $\tau$ identifying the threshold, and $\lambda$ the non-centrality parameter defined as

$$
\lambda(\boldsymbol{V})=\operatorname{vec}\left(\boldsymbol{V}-\boldsymbol{V}_{0}\right)^{\mathrm{T}} \boldsymbol{H}^{\mathrm{T}} \boldsymbol{C}^{-1} \boldsymbol{H} \operatorname{vec}\left(\boldsymbol{V}-\boldsymbol{V}_{0}\right) .
$$

The case where $\boldsymbol{H}$ is ill-conditioned is also addressed in [8] by means of the Singular Value Decomposition (SVD) [16].

\footnotetext{
${ }^{1}$ This means that the number of sections $N$ and the sequence of the time intervals are known parameters. Instead, the case where such parameters are unknown is fully provided in [8]. However, nothing changes for the scope of the proposed optimization problem since it does not depend on the detection strategy.
} 


\section{Optimization Problem Formulation}

In this section, a route planning strategy able to optimally determine the ship trajectory is formalized, and an efficient technique is proposed to handle the resulting non-convex optimization problem. The main tasks and objectives to account for at the design stage are the following:

- the vessel meets and fulfills its mission, i.e., reaches a specific point $\boldsymbol{p}^{\star}$, where a rendezvous with another ship (for some possible illegal exchanges) will occur;

- vessel kinematics satisfies some physical constraints and requirements;

- the anomaly trajectory is as hidden as possible, namely the detection capabilities of the sensing system are minimized.

Moreover, the temporal scale parameters characterizing the piecewise OU model are assumed to be all equal without loss of generality, i.e., $\Delta t=\left[t_{n}, t_{n-1}\right], \forall n=1, \ldots, N$, which is set up so that the process reaches a steady state behavior in each time interval. Thus, the degrees of freedom available at the route planning stage are given by

- the $N$ vessel mean velocity vectors stacked in matrix $\boldsymbol{V}$, characterizing the ship ensemble behavior;

- the $N+1$ vessel positions comprised in matrix $\boldsymbol{P}$.

\section{A. Route design problem, figure of merit, and constraints}

The route planning problem aimed at optimally defining the ship positions as well as the underlying OU random process parameters, can be formulated as the following constrained optimization problem.

$$
\mathcal{P} \begin{cases}\min _{\boldsymbol{V}, \boldsymbol{P}} & \max _{i=1, \ldots, K} \lambda_{i}(\boldsymbol{V}) \\ \text { s.t. } & \left\|\boldsymbol{v}_{h}\right\| \leq v_{\max }, h=1, \ldots, N \\ & \left\|\boldsymbol{v}_{h}-\boldsymbol{v}_{h-1}\right\| \leq a_{\max }, h=2, \ldots, N \\ & \left\|\boldsymbol{p}_{h}-\left(\boldsymbol{v}_{h} \Delta t+\boldsymbol{p}_{h-1}\right)\right\|_{\boldsymbol{C}^{-1}}^{2} \leq \epsilon, h=1, \ldots, N \\ & \min \quad \max \left(\left\|\boldsymbol{p}_{k}-\boldsymbol{p}^{\star}\right\|\right) \leq \epsilon_{1} \\ & \boldsymbol{V}=\left[\boldsymbol{v}_{1}, \boldsymbol{v}_{2}, \ldots, \boldsymbol{v}_{N}\right] \in \mathbb{R}^{2, N} \\ & \boldsymbol{P}=\left[\boldsymbol{p}_{0}, \boldsymbol{p}_{1}, \boldsymbol{p}_{2}, \ldots \boldsymbol{p}_{N}\right] \in \mathbb{R}^{2, N+1} \\ & \boldsymbol{p}_{h} \in \mathcal{S}_{c}, h=1, \ldots, N \\ & \boldsymbol{p}_{0}=\mathbf{0}\end{cases}
$$

Specifically, the performance metric considered is

$$
\lambda(\boldsymbol{V})=\max _{i=1, \ldots, K} \lambda_{i}(\boldsymbol{V}),
$$

where

- $\lambda_{i}(\boldsymbol{V})=\operatorname{vec}\left(\boldsymbol{V}-\boldsymbol{V}_{0}\right)^{\mathrm{T}} \boldsymbol{H}_{i}^{\mathrm{T}} \boldsymbol{C}_{i}^{-1} \boldsymbol{H}_{i} \operatorname{vec}\left(\boldsymbol{V}-\boldsymbol{V}_{0}\right)$, $i=1, \ldots, K$, is the non-centrality parameter associated with the $i$-th detector, which exploits the subset $\mathcal{S}_{i}$;

- $\mathcal{S}_{i}=\left\{t_{i, 1}^{m}, \ldots, t_{i, K_{i}}^{m}\right\}$ is the subset of time instants where measurements can be collected, with $K_{i}=\left|\mathcal{S}_{i}\right| \leq$ $K \leq N$ the cardinality of the subset $\mathcal{S}_{i}, t_{i, h}^{m} \in$ $\left\{t_{1}^{m}, t_{2}^{m}, \ldots, t_{K}^{m}\right\}, h=1, \ldots, K_{i}$, and $t_{i, h}^{m} \neq t_{i, k}^{m}, h \neq k$;

- $\boldsymbol{H}_{i}$ and $\boldsymbol{C}_{i}$ depend on the available measurements (see equations (12) and (13), respectively);
- $K$ is the number of possible detectors that the surveillance system may implement as function of the available measurements.

- $\mathcal{S}_{c} \subseteq \mathbb{R}^{2}$ is a subset of the two-dimensional space, that is assumed convex for mathematical tractability. It can be described by means of polyhedra, ellipsoids, as well as their intersection to appropriately model coast impairments.

In particular, $\lambda_{i}(\boldsymbol{V})$ is proportional to the Kullback-Leibler (KL)-divergence between the actual distributions under the $H_{0}$ and $H_{1}$ hypotheses. Specifically, denoting by $f_{1}\left(\boldsymbol{y}_{i}\right)$ and $f_{0}\left(\boldsymbol{y}_{i}\right)$ the distribution of the observation $\boldsymbol{y}_{i}$ collected at time instants $\mathcal{S}_{i}$ under the $H_{0}$ and $H_{1}$ hypotheses, respectively, it follows that [17]

$$
\lambda_{i}(\boldsymbol{V})=2 \mathcal{D}_{\mathrm{KL}}\left(f_{1}\left(\boldsymbol{y}_{i}\right) \mid f_{0}\left(\boldsymbol{y}_{i}\right)\right), \quad i=1, \ldots, K .
$$

As a consequence, since KL-divergence controls the performance limits for any given hypothesis testing problem [17], the non-centrality parameter (associated with a given subset of measurements time instants $\mathcal{S}_{i}$ ) represents a viable means to predict the sensing system's surveillance capabilities. It is also worth observing that the detection performance of the clairvoyant receiver, obtained assuming known the ship velocity profile under the two hypotheses, is dependent on $\lambda_{i}(\boldsymbol{V})$ too. As a result, controlling $\lambda_{i}(\boldsymbol{V})$ allows to rule an upper bound to the surveillance system performance. In conclusion, $\lambda(\boldsymbol{V})$ provides a meaningful performance measure of the ship detectability.

Finally, regarding the conditions in Problem $\mathcal{P}$, the first two constraints account for the physical requirements on the mean velocity dynamic, the third constraint guarantees the compliance of the trajectory with the underlying statistical model, the fourth constraint ensures the existence of a time instant allowing the rendezvous, the fifth constraint controls sea coast impairments, and the last one defines the starting point of the vessel trajectory.

\section{B. Efficient optimization technique}

Due to the stopping area constraint, i.e.,

$$
\min _{h=1, \ldots, N-1} \max _{k=h, \ldots, h+L}\left(\left\|\boldsymbol{p}_{k}-\boldsymbol{p}^{\star}\right\|\right) \leq \epsilon_{1}
$$

Problem $\mathcal{P}$ turns out to be a non-convex optimization problem (and in general hard to solve). However, it is possible to prove its hidden convexity. Specifically a global optimal solution to the formulated route planning problem can be obtained via an efficient solution technique briefly summarized in Algorithm 1. Therein, $\mathcal{P}_{l}, l=1, \ldots, N-L+1$, is the convex optimization 


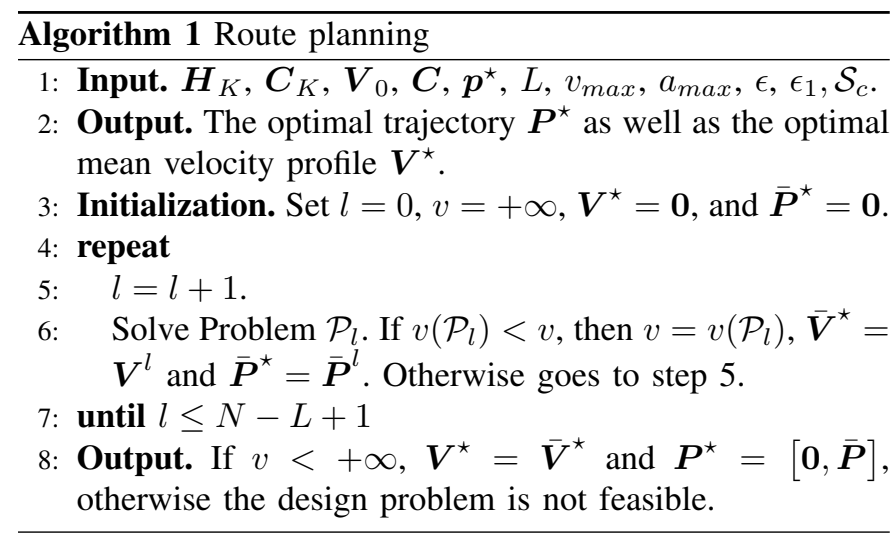

problem, defined as follows.

$\mathcal{P}_{l} \begin{cases}\min _{\boldsymbol{V}, \overline{\boldsymbol{P}}} & \lambda(\boldsymbol{V}) \\ \text { s.t. } & \left\|\boldsymbol{v}_{h}\right\| \leq v_{\max }, h=1, \ldots, N \\ & \left\|\boldsymbol{v}_{h}-\boldsymbol{v}_{h-1}\right\| \leq a_{\max }, h=2, \ldots, N \\ & \left\|\boldsymbol{p}_{h}-\boldsymbol{v}_{h} \Delta t\right\|_{\boldsymbol{C}^{-1}} \leq \sqrt{\epsilon}, h=1, \ldots, N \\ & \left\|\boldsymbol{p}_{h}-\left(\boldsymbol{v}_{h} \Delta t+\boldsymbol{p}_{h-1}\right)\right\|_{\boldsymbol{C}^{-1}} \leq \sqrt{\epsilon}, h=2, \ldots, N \\ & \max \left(\left\|\boldsymbol{p}_{k}-\boldsymbol{p}^{\star}\right\|\right) \leq \epsilon_{1} \\ & k=l, \ldots, l+L \\ & \boldsymbol{V}=\left[\boldsymbol{v}_{1}, \boldsymbol{v}_{2}, \ldots, \boldsymbol{v}_{N}\right] \in \mathbb{R}^{2, N} \\ & \overline{\boldsymbol{P}}=\left[\boldsymbol{p}_{1}, \boldsymbol{p}_{2}, \ldots, \boldsymbol{p}_{N}\right] \in \mathbb{R}^{2, N} \\ & \boldsymbol{p}_{h} \in \mathcal{S}_{c}, h=1, \ldots, N,\end{cases}$

where

$$
\lambda(\boldsymbol{V})=\operatorname{vec}\left(\boldsymbol{V}-\boldsymbol{V}_{0}\right)^{\mathrm{T}} \boldsymbol{H}_{K}^{\mathrm{T}} \boldsymbol{C}_{K}^{-1} \boldsymbol{H}_{K} \operatorname{vec}\left(\boldsymbol{V}-\boldsymbol{V}_{0}\right)
$$

directly refers to the non-centrality parameter associated to the maximum number of measurements. For any $l=1, \ldots, N-$ $L+1, \mathcal{P}_{l}$ is a convex optimization problem and the optimal solution to $\mathcal{P}$ is $\left(\boldsymbol{V}^{\star}, \boldsymbol{P}^{\star}\right)$, with

$$
\begin{aligned}
& \boldsymbol{V}^{\star}=\boldsymbol{V}^{l^{\star}} \\
& \boldsymbol{P}^{\star}=\left[\mathbf{0}, \overline{\boldsymbol{P}}^{l}\right],
\end{aligned}
$$

where

$$
l^{\star}=\arg \min _{l=1, \ldots, N-L+1} \lambda\left(\boldsymbol{V}^{l}\right),
$$

and $\left(\boldsymbol{V}^{l}, \overline{\boldsymbol{P}}^{l}\right)$ is an optimal solution to $\mathcal{P}_{l}$. Note that, if $\mathcal{P}_{l}$ is not feasible, the corresponding optimal value is assumed equal to $+\infty$.

The computational complexity related to the implementation of Algorithm 1 depends on the number of outer iterations $N-$ $L+1$, as well as on the complexity of each iteration. Precisely, the overall complexity is linear with respect to $N$ while, the complexity of each cycle is that required to solve (for our specific applications) a Second-Order Cone Program (SOCP), which is $O\left(N^{3.5} \log (\eta)\right)$ (see [18]), where $\eta$ is a prescribed accuracy.

The route design problem, all the related route constraints, and the proposed solution will be fully explained and detailed in the extended version of this work.

\section{Performance ANAlysis of REAL-WORLD VESSEL TRAFFIC DATA}

The optimization strategy proposed in this work has been applied to the real-world AIS track of a vessel navigating in the waters of the Pacific Ocean [19], which was already analyzed in [8] and [9]. The information provided by the track reveals a deviation from the nominal route, depicted in Fig. 1 by the black dashed line (ground truth), in order to rendezvous with other ships in the specified point $\boldsymbol{p}^{*}$.

To test the proposed strategy, a gap in AIS data of about $T=145.5 \mathrm{~h}$ was artificially introduced to simulate the AIS device shutdown during the actual deviation. Additionally, a fictive nominal trajectory (blue dotted curve) has been assumed when the data gap occurs, which is characterized by a piecewise nominal velocity $\boldsymbol{V}_{0} \in \mathbb{R}^{2, N}$, with $N=37$, and whose mean value is $\boldsymbol{v}_{0}=[3.36,-0.42]^{\mathrm{T}} \mathrm{m} / \mathrm{s}$. The other OU parameters are also estimated on this fictive nominal trajectory, and they are given by $\gamma=\left[1.63 \times 10^{-5}, 5.95 \times 10^{-4}\right]$ and $\boldsymbol{\sigma}=\left[1.1 \times 10^{-2}, 1.58 \times 10^{-2}\right]$.

As regards the optimization procedure, the parameters related to the constraints are set as: $v_{\max }=10 \mathrm{~m} / \mathrm{s}, a_{\max }=1$, $\epsilon=1$, and $\epsilon_{1}=1 \mathrm{~km}$. Moreover, an ellipse constraint, which can indicate the presence of land (or forbidden navigational area), has been imposed to contain $\boldsymbol{p}^{*}$ and the resulting optimized trajectory, and it has been designed by aligning the focal points with the positions where the AIS device is turned off and where is then reactivated, respectively.

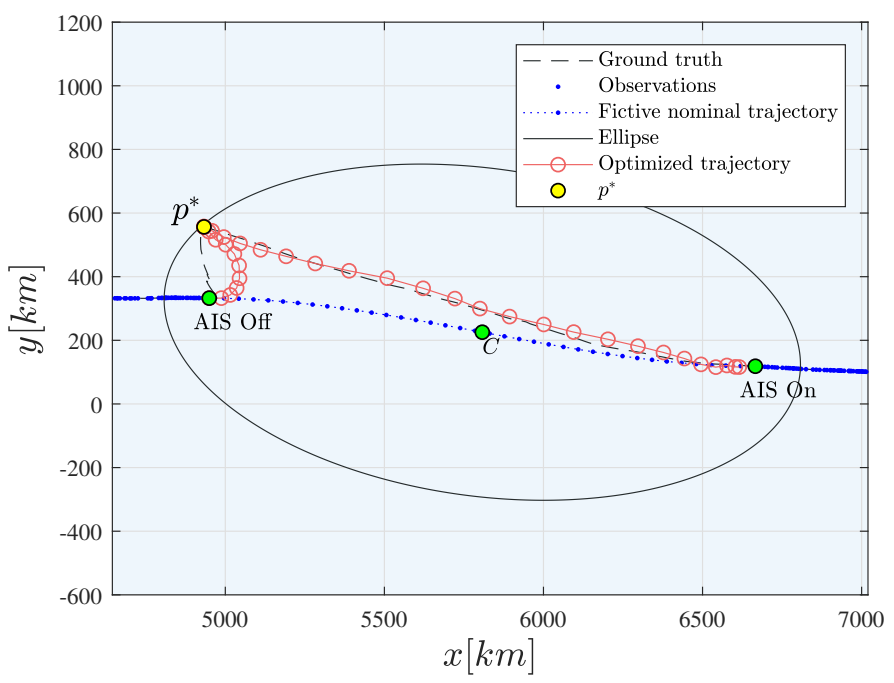

Fig. 1. Real-world AIS track (black dotted line) versus optimized trajectory (green o-marker curve).

As it can be visually noticed in Fig. 1, the optimized trajectory is effectively devised (red o-marker curve) so that the vessel can arrive to the meeting point, loiter there for the desired time $(L \Delta t \simeq 19.6 \mathrm{~h}$ ), and finally restore the nominal condition before the AIS device reactivation.

Performance of detector (14) is provided in terms of missed detection probability, $1-P_{\mathrm{D}}$, versus false alarm probability, 


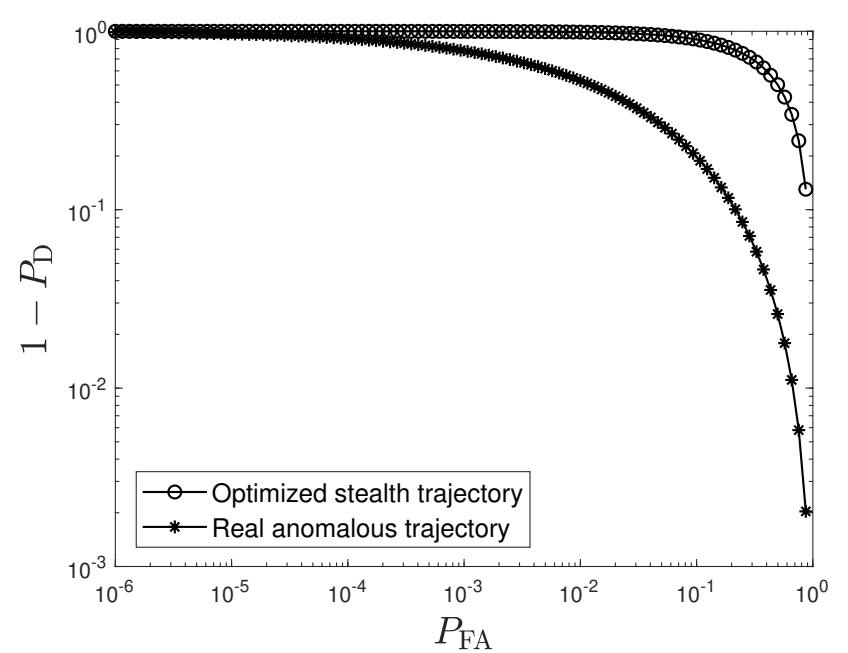

Fig. 2. Performance of anomaly detector (14) shows the optimized trajectory being less detectable then the real one.

$P_{\mathrm{FA}}$, in Fig. 2, where the ROC curves related to the real trajectory (star-marker curve) and the optimized one (o-marker curve) are shown. Remarkably, the synthesized stealth trajectory exhibits a behavior similar to the real anomalous route, but it turns out to be stealthier, revealing the effectiveness of the new proposed route planning tool.

\section{Conclusions}

The worst conditions in terms of anomaly detection capability are investigated proposing an optimization methodology to design a vessel trajectory as stealth as possible. Such trajectory is formalized as a min-max problem which exploits the KLdivergence between the two statistical hypotheses (nominal versus anomalous) as key performance measure. Considering the OU mean-reverting stochastic process as the vessel's dynamic model, proper constraints at the design stage are forced accounting for physical, practical, and kinematic requirements. The resulting non-convex optimization problem is solved via a computationally efficient strategy, and a real-world case study is reported to assess the effectiveness of the proposed strategy.

\section{ACKNOWLEDGMENT}

This work was supported by the NATO Allied Command Transformation (ACT) via the project Data Knowledge Operational Effectiveness (DKOE) at the NATO Science and Technology Organization (STO) Centre for Maritime Research and Experimentation (CMRE). The authors thankfully acknowledge MarineTraffic and Prof. Dimitris Zissis from the University of the Aegean for providing the real-world AIS data set used for the experimental analysis and for the valuable domain expert insights.

\section{REFERENCES}

[1] "International Convention of Safety of Life at Sea (SOLAS)," International Maritime Organization (IMO).
[2] K. Kowalska and L. Peel, "Maritime anomaly detection using Gaussian process active learning," in 2012 15th International Conference on Information Fusion, July 2012, pp. 1164-1171.

[3] M. Vespe, I. Visentini, K. Bryan, and P. Braca, "Unsupervised learning of maritime traffic patterns for anomaly detection," in 9th IET Data Fusion Target Tracking Conference (DF TT 2012): Algorithms Applications, May 2012, pp. 1-5.

[4] M. Riveiro, G. Pallotta, and M. Vespe, "Maritime anomaly detection: A review," Wiley Interdisciplinary Reviews: Data Mining and Knowledge Discovery, vol. 8, p. e1266, 052018.

[5] B. Ristic, B. L. Scala, M. Morelande, and N. Gordon, "Statistical analysis of motion patterns in AIS data: Anomaly detection and motion prediction," in 2008 11th International Conference on Information Fusion, June 2008, pp. 1-7.

[6] R. O. Lane, D. A. Nevell, S. D. Hayward, and T. W. Beaney, "Maritime anomaly detection and threat assessment," in 2010 13th International Conference on Information Fusion, July 2010, pp. 1-8.

[7] F. Katsilieris, P. Braca, and S. Coraluppi, "Detection of malicious AIS position spoofing by exploiting radar information," in Proceedings of the 16th International Conference on Information Fusion, July 2013, pp. 1196-1203.

[8] E. d'Afflisio, P. Braca, L. M. Millefiori, and P. Willett, "Detecting anomalous deviations from standard maritime routes using the Ornstein-Uhlenbeck process,' IEEE Transactions on Signal Processing, vol. 66, no. 24, pp. 6474-6487, Dec 2018.

[9] E. d'Afflisio, P. Braca, L. M. Millefiori, and P. Willett, "Maritime anomaly detection based on mean-reverting stochastic processes applied to a real-world scenario," in 2018 21st International Conference on Information Fusion (FUSION), July 2018, pp. 1171-1177.

[10] L. M. Millefiori, P. Braca, K. Bryan, and P. Willett, "Modeling vessel kinematics using a stochastic mean-reverting process for long-term prediction," IEEE Transactions on Aerospace and Electronic Systems, vol. 52, no. 5, pp. 2313-2330, October 2016.

[11] L. M. Millefiori, P. Braca, and P. Willett, "Consistent estimation of randomly sampled Ornstein-Uhlenbeck process long-run mean for longterm target state prediction." IEEE Signal Processing Letters, vol. 23, no. 11, pp. 1562 - 1566, November 2016.

[12] G. Vivone, L. M. Millefiori, P. Braca, and P. Willett, "Performance assessment of vessel dynamic models for long-term prediction using heterogeneous data," IEEE Transactions on Geoscience and Remote Sensing, vol. 55, no. 11, pp. 6533-6546, November 2017.

[13] M. Uney, L. M. Millefiori, and P. Braca, "Prediction of rendezvous in maritime situational awareness," in 2018 21st International Conference on Information Fusion (FUSION), July 2018, pp. 622-628.

[14] X. R. Li and V. P. Jilkov, "Survey of maneuvering target tracking. Part I. Dynamic models," IEEE Transactions on Aerospace and Electronic Systems, vol. 39, no. 4, pp. 1333-1364, Oct 2003.

[15] P. Coscia, P. Braca, L. M. Millefiori, F. Palmieri, and P. Willett, "Maritime traffic representation based on sea-lanes graph construction criteria using multiple Ornstein-Uhlenbeck processes," IEEE Transactions on Aerospace and Electronic Systems, to be published, 2018.

[16] A. Thorpe and L. Scharf, "Data adaptive rank-shaping methods for solving least squares problems," IEEE Transactions on Signal Processing, vol. 43, no. 7, pp. 1591-1601, july 1995 .

[17] T. M. Cover and J. A. Thomas, Elements of information theory. John Wiley \& Sons, 2012.

[18] A. Ben-Tal and A. S. Nemirovskiaei, Lectures on Modern Convex Optimization: Analysis, Algorithms, and Engineering Applications. Philadelphia, PA, USA: Society for Industrial and Applied Mathematics, 2001.

[19] J. J. Alava, M. J. Barragán-Paladines, J. Denkinger, L. Muñoz-Abril, P. Jiménez, F. Paladines, and et al., "Massive Chinese fleet jeopardizes threatened shark species around the Galápagos marine reserve and waters off Ecuador: Implications for national and international fisheries policy," International Journal of Fisheries Sci Res., 2017. 